\title{
PRÉSENTATION DU RAPPORT
}

\section{Indications préliminaires}

Il semble opportun d'indiquer tout d'abord ce sur quoi porte le rapport, ce qu'il ne contient pas et la manière dont il a été structuré. Plusieurs points sont à ce sujet à préciser :

- le rapport porte essentiellement sur le fonctionnement biogéochimique de la biosphère terrestre; il contient cependant quelques données générales sur le milieu océanique, qui naturellement est en interaction avec le domaine continental. Il traite aussi dans certains cas des interactions entre la biosphère et le système géologique ;

- il s'intéresse plus particulièrement aux relations "sol-plante », donc au sol et à ses composants; de ce fait, il n'aborde pas les problèmes plus spécifiques liés à la croissance végétale qui relèvent de l'écophysiologie fonctionnelle et de la bioclimatologie.

Ceci indiqué, le rapport comprend quatre parties, qui peuvent être regroupées en deux grands ensembles.

- Le premier ensemble (1 ${ }^{\text {ère }}$ et $2^{\mathrm{e}}$ parties) traite, en s'appuyant sur quelques exemples caractéristiques, des cycles biogéochimiques à proprement parler et fait le point sur les processus mis en jeu au cours des recyclages. Il est abordé, soit par éléments (1 ${ }^{\text {rè }}$ partie) en prenant en compte tous les compartiments où ceux-ci sont susceptibles d'intervenir, soit par milieux ( $2^{\mathrm{e}}$ partie), c'est-à-dire en portant son attention sur un compartiment donné et en s'intéressant aux différents éléments qui peuvent y jouer un rôle.

- Le second ensemble ( $3^{e}$ et $4^{e}$ parties) regroupe une série de points tournant autour de la nécessaire amélioration de nos connaissances sur les cycles, surtout si l'on veut obtenir d'eux un maximum de services. Il a trait d'abord à un certain nombre d'actions de recherche disciplinaires sur lesquelles il est nécessaire d'obtenir un effort appuyé. II concerne ensuite des actions d'ordre multidisciplinaire qui, s'appuyant sur des modélisations et des observations, permettront $d$ 'avancer dans la gestion de ces systèmes biogéochimiques complexes. 


\section{2 | Approche par éléments (1 ère partie)}

La première partie traite du comportement d'un certain nombre d'éléments qui sont apparus à l'analyse comme étant à la base du fonctionnement biogéochimique de la biogéosphère (naturelle et anthropisée). Elle comprend quatre chapitres:

- Le chapitre 1 est consacré à la présentation du cycle du carbone, qui constitue l'élément majeur de l'histoire générale de la biogéosphère, mais qui « bien que le plus étudié est encore insuffisamment connu »'. En effet cet élément intervient à tous les niveaux : différents compartiments (atmosphère-sols terrestres-sédiments marins), différents états chimiques $\left(\mathrm{CO}_{2}\right.$-composés organiques), différentes formes (êtres vivants-carbonatescombustibles fossiles), différentes échelles de temps (depuis l'année pour les processus biologiques jusqu'à plusieurs centaines de millions d'années pour l'altération des roches et la fossilisation de la matière organique). II a de ce fait une dimension géologique incontestable, mais en outre, c'est un élément qui est beaucoup lié à l'anthropisation en raison de la très forte redistribution que l'homme lui fait subir en relation avec ses besoins énergétiques en combustibles fossiles; ceci sans oublier le rôle majeur que le carbone joue à la surface du globe sur le climat (effet de serre), ainsi qu'à travers la fabrication grâce à la photosynthèse d'un grand nombre de molécules carbonées, à laquelle participe naturellement la production agricole. Ces derniers développements assurent la transition avec les éléments étudiés dans le chapitre 2.

- Le chapitre 2 concerne l'azote, le phosphore et le soufre qui sont abordés ensemble du fait que ces nutriments majeurs des organismes phototrophes (végétaux) peuvent avoir en même temps des effets négatifs sur l'environnement (ce qui n'est pas le cas d'un autre nutriment majeur, tel le potassium); par ailleurs tous les trois font intervenir des processus généraux communs, contrôlés en totalité ou en partie par les communautés microbiennes du sol. Pour ces trois éléments, des cycles sensiblement équilibrés, basés sur la mise en œuvre de systèmes tampons naturels qui les régulaient, avaient été mis en place au cours de l'évolution de la biosphère. Il $\mathrm{n}^{\prime}$ en est plus de même aujourd'hui en raison de l'anthropisation accélérée de la planète et notamment de l'intensification des activités agricoles depuis une cinquantaine d'années. Au cours de ce chapitre, il est fait état de l'apparition de dysfonctionnements des cycles de ces nutriments, ainsi que de la détérioration des services écosystémiques à en attendre. L'approche sera présentée par éléments, mais en réalité ce sont déjà les problèmes

'B. Tissot et al. (2005). Perspectives énergétiques. Académie des sciences, DNBR, tome VIII. 
des agrosystèmes modernes, en tant que milieux, qui sont évoqués dans ce chapitre.

- Le chapitre 3 traite des cycles biogéochimiques du silicium, de l'aluminium et du fer qui sont, après l'oxygène, les trois éléments majeurs de la croûte terrestre (tableau 3.1). Ils sont à la base de l'évolution géochimique de la lithosphère (altération des roches et érosion chimique des terres émergées) (cf. figure 1.4.) dont les caractéristiques générales sont bien connues, à savoir une redistribution notable de la silice, alors que l'aluminium et le fer (avec les éléments en traces métalliques qui l'accompagnent) sont très peu redistribués. Mais ce chapitre traite aussi des aspects moins connus concernant l'action de ces éléments en relation avec le développement des êtres vivants où ils peuvent, dans un certain nombre de situations, jouer le rôle de facteur limitant (ceci est très net dans les océans notamment pour le silicium et le fer). Le recyclage biologique de la silice, qui arrive à contrôler dans certains cas la géochimie des sols, la phytotoxicité de l'aluminium et enfin l'influence du fer sur l'activité microbienne sont spécialement analysés.

- Enfin, le chapitre 4 s'intéresse aux radionucléides, en raison de leur impact sur l'environnement (IRSN, 1994), mais qui avaient été peu intégrés jusque-là dans le cadre d'une vision globale des échanges, fondement même de la conception biogéochimique. Ceci est particulièrement net pour les radionucléides artificiels produits par la fission nucléaire, dont la mise en œuvre ne date que de quelques décennies. Des connaissances approfondies dans le domaine de la spéciation s'avèrent indispensables pour ces éléments, ainsi d'ailleurs que la connaissance de leurs interactions avec les activités biologiques et notamment avec les micro-organismes.

\section{Approche par milieux (2 $2^{\mathrm{e}}$ partie)}

Si, dans la première partie, l'analyse des cycles biogéochimiques est envisagée globalement par élément ou groupe d'éléments, la deuxième partie aborde le problème par milieux en considérant simultanément l'ensemble des éléments concernés dans le fonctionnement d'un écosystème donné ; l'écosystème, constitué par les interactions fonctionnelles biocénose-biotope étant considéré comme l'élément de base de la biosphère. Tous les milieux ne pouvant être examinés dans ce rapport, le choix retenu porte sur cinq d'entre eux qui vont servir de références à l'extension des connaissances à d'autres écosystèmes; deux concernent les milieux semi-naturels ou encore très peu artificialisés : domaine continental forestier et domaine océanique. Les trois autres s'intéressent ensuite à des milieux particuliers, qui sont très artificialisés par suite d'une intense activité 
anthropique : d'abord les agrosystèmes animaux notablement perturbés dans le cadre d'une activité industrialisée en relation avec la forte production d'effluents d'élevage (matières organiques exogènes), ensuite des milieux fortement modifiés du fait de l'activité minière, industrielle ou urbaine, enfin le domaine estuarien représentant des milieux littoraux semi-fermés, généralement à haute productivité, mais où viennent se déverser de nombreux rejets en rapport avec les interventions humaines des dernières décennies.

- Le chapitre 5, consacré aux écosystèmes peu anthropisés, aborde l'étude de deux grands domaines de la surface du globe, qui sont naturellement liés, mais qui fonctionnent différemment : d'une part, les écosystèmes forestiers en tant que modèles des écosystèmes continentaux et d'autre part les océans, qui jouent un rôle majeur dans le cycle du $\mathrm{CO}_{2}$.

- Les écosystèmes forestiers (5.1) ont été retenus d'abord parce qu'ils sont très représentatifs des milieux terrestres naturels (ils couvrent encore $22 \%$ des terres émergées et fournissent $50 \%$ environ de la production primaire de la planète) ; mais ils sont intéressants aussi du fait qu'ils constituent un bon exemple de type de milieux à sols pauvres où, sans intrants, sans pratiques culturales et sans traitement particulier mais grâce à des recyclages biogéochimiques performants, ils sont à même d'assurer de façon régulière une production de biomasse (certes ligneuse) très intéressante, tout en jouant un rôle écologique et environnemental de premier plan (cycle de l'eau, stockage du carbone, impact paysager, ...). De plus, de tels écosystèmes autonomes et efficaces sont le prototype même des agrosystèmes extensifs (opposés à ceux dont il est question dans le chapitre 5.2), donc se rapprochant d'un mode d'agriculture biogéochimiquement contrôlée, base dans le domaine agricole de ce que l'on appelle aujourd'hui le « développement durable».

La présentation concerne essentiellement les écosystèmes gérés de la zone tempérée, car à la suite des phénomènes de dépérissement dans les années 1980 (pluies acides), ceux-ci ont donné lieu à une série de recherches qui peuvent de ce fait servir de modèles à l'étude biogéochimique de beaucoup d'autres écosystèmes terrestres : forêts tropicales, prairies naturelles...

- Le sous-chapitre 5.2 expose les problèmes généraux concernant les milieux océaniques et décrit les espoirs qui sont attendus du développement de la biogéochimie marine (par exemple : les facteurs limitants de la nutrition, tels le silicium, le fer, le phosphore, ... n'étant pas toujours ce que l'on attendait). Dans ce domaine, les questions à résoudre restent encore nombreuses et spécifiques malgré les travaux déjà réalisés dans le cadre du GIEC : évaluation des interactions 
avec l'atmosphère (climat-transport intercontinental de matière, ...) ainsi que du rôle des sédiments, importance des phénomènes biologiques non encore bien explorés concernant notamment les populations planctoniques, insuffisance dans la connaissance des processus hydrodynamiques qui sont à l'origine des circulations océaniques et des phénomènes de stratification. Tout ceci fait qu'on se trouve aujourd'hui devant un grand chantier exigeant la présence de nombreux réseaux d'observations et de mesures biogéochimiques dont il sera fait état au chapitre 9.

- Le chapitre 6 s'attache à présenter le fonctionnement biogéochimique de quelques milieux où se manifeste une pression anthropique tout à fait spécifique. Trois cas ont été retenus :

- Le premier (sous-chapitre 6.1) passe en revue les problèmes relatifs à l'épandage sur les terres agricoles des matières organiques exogènes (MOE) dont $95 \%$ en France sont représentés par des effluents, en provenance de zones d'élevages intensifiés et impliquant de ce faif un apport exogène d'aliments du bétail. La question abordée est la suivante : comment tirer le meilleur parti de ces $M O E$, qui sont à la fois des amendements et des engrais organiques, et comment s'appuyer sur les recyclages biogéochimiques pour empêcher l'individualisation de "zones d'excédent structurel » susceptibles de présenter des phénomènes de pollution de l'environnement : air (émissions gazeuses de composés azotés), eaux (azote, phosphore, pesticides) et sols (zinc, cuivre, phosphore et autres éléments en traces métalliques). Des indications à propos de la régulation et du contrôle sont apportées dans ce domaine, d'une part grâce à la prévention résultant $d$ 'une meilleure gestion de l'alimentation animale, $d$ 'autre part grâce à l'aide de traitements (fermenteur), et enfin en s'appuyant sur un raisonnement agronomique reposant sur une gestion réfléchie des apports aux sols.

- Le deuxième (sous-chapitre 6.2) traite avant tout de la perturbation des cycles dans les sites miniers et industriels qui sont très particuliers du fait que la redistribution des éléments métalliques sart du schéma classique des systèmes naturels. Il est donc souhaitable d'envisager ce genre de problèmes d'environnement en introduisant la notion de cycle biogéochimique, ne serait-ce que pour s'extraire du cadre habituel «pollution-effet».

- Le troisième cas (sous-chapitre 6.3) concerne le comportement des contaminants chimiques, organiques et métalliques, dans les estuaires - zones parmi les plus productives du domaine marin qui constituent des interfaces particulières entre continents et océans, donc à la fois solidaires du bassin versant du fleuve, mais aussi en 
continuité avec les systèmes côtiers. Soumis plus que d'autres écosystèmes marins aux effets de l'activité humaine, ils sont le siège de piégeages et de recyclages des éléments de grande intensité, dont la maîtrise passe par une bonne connaissance des processus biogéochimiques mis en jeu.

\section{Secteurs et disciplines à renforcer ( $3^{e}$ partie)}

La troisième partie insiste sur deux secteurs de recherche sur lesquels des efforts doivent être réalisés en vue d'une meilleure connaissance des cycles biogéochimiques:

- un renforcement des études sur les micro-organismes et l'écologie microbienne des sols;

- un approfondissement dans le domaine des matières organiques des sols (nature et réactivité).

D'autres secteurs scientifiques, qui sont aussi très importants en vue d'une meilleure compréhension des cycles biogéochimiques, n'ont pas pu être analysés dans cette partie du rapport. II s'agit notamment de l'action de la faune tellurique et surtout de la spéciation des éléments chimiques, éléments en traces compris. Ils n'ont pas pour autant été oubliés, mais ont été développés ailleurs dans le document:

- pour la faune dans les chapitres 2 et 7 ;

- pour la spéciation dans les chapitres 4 et 10 pour les radionucléides, dans les chapitres 3 et 7 pour l'aluminium et le fer et dans le chapitre 7 pour le phosphore.

- Le chapitre 7 traite du rôle joué par les organismes vivants décomposeurs (saprophytes), essentiellement des micro-organismes (bactéries et champignons notamment) dans le recyclage des éléments au sein de la biosphère de surface et des sols en particulier là titre d'exemple $90 \%$ environ du $\mathrm{CO}_{2}$ relargué par les écosystèmes résultent de leur activité). Un tel recyclage découle avant tout de leur action, d'une part dans l'incorporation de nombreux éléments par la matière vivante (biosynthèse) et dans la dégradation des substances organiques naturelles ou synthétiques (biodégradation), d'autre part dans la décomposition des minéraux (géomicrobiologie); mais aussi du fait qu'ils agissent comme agents (directs ou indirects) dans les 
réactions d'oxydation, réduction ou complexation d'éléments majeurs (carbone, azote, phosphore, soufre) et traces (fer, manganèse, cuivre, ...).

Ceci étant, il est bon d'indiquer que de nombreux verrous technologiques demeurent aujourd'hui; aussi bien pour identifier cette microflore tellurique que pour préciser la nature des communautés microbiennes, en particulier la microflore rhizosphérique, ou encore pour appréhender les nombreuses stratégies énergétiques et nutritionnelles mises en jeu. C'est donc une véritable « biogéochimie microbienne » qu'il s'agit maintenant de développer.

Une note dans ce chapitre est en outre consacrée à une brève présentation de la «biosphère souterraine profonde », dont l'existence n'a été détectée que récemment et dont l'impact sur les grands cycles biogéochimiques est encore à ce jour peu connu. II est clair pourtant que ce type de métabolisme devrait à l'avenir être pris en considération, ne serait-ce que dans les projets de séquestration souterraine du $\mathrm{CO}_{2}$ pour lutter contre les gaz à effet de serre.

- Le chapitre 8 concerne les matières organiques des milieux supergènes, c'est-à-dire l'ensemble des composés carbonés d'origine biologique, qui constitue le plus grand stock labile de nutriments, tels azote, phosphore et soufre, dans les sols, les sédiments et les eaux et qui joue de ce fait un rôle clé en biogéochimie. Il traite surtout de la nature et des fonctions de la matière organique des sols, ces derniers servant, à la surface de notre planète, $d$ 'intermédiaires privilégiés entre constituants minéraux et matière vivante. Toute une recherche doit être entreprise pour mieux définir les propriétés chimiques, physicochimiques et biogéochimiques de ces macromolécules à structure aléatoire, qui forment d'ailleurs un véritable continuum entre les composés les plus simples et les gros polycondensats. C'est là un travail très délicat, car il implique au préalable la mise au point de méthodes préparatoires évitant toute dénaturation.

\section{Aspects méthodologiques, observations et modélisations ( $4^{\mathrm{e}}$ partie)}

L'examen des cycles biogéochimiques, qui sont présentés au cours de la première et de la deuxième partie, montre sans ambiguité que ceux-ci reposent sur le comportement de nombreux éléments susceptibles d'intervenir dans des contextes à la fois hétérogènes, non stationnaires, spatialisés et aussi à différentes échelles temporelles; d'où la nécessité de s'investir dans des travaux de 
modélisation biogéochimique à partir de données dont il faut auparavant assurer la collecte.

Dans ces conditions, toute étude à ce sujet comporte deux phases :

- l'obtention de résultats numériques fiables;

- le traitement des données et la conception de modèles.

Ces points constituent la charpente des deux chapitres de cette quatrième partie, tout en précisant qu'il ne s'agit pas de les opposer, mais plutôt d'arriver à les fusionner au sein d'un seul dispositif conceptuel qui sert de principe unificateur. La collaboration doit donc se faire dans les deux sens : naturellement, des données expérimentales aux modèles, mais aussi des modèles aux données prévisionnelles; ces dernières devant être sans cesse recalées en fonction de l'avancement de la modélisation, de l'acquisition de nouvelles données et de l'évolution des questions posées. C'est la raison pour laquelle le rapport présente d'abord les opérations de modélisation (chapitre 9) et qu'il traite ensuite du problème des observatoires et des réseaux (chapitre 10).

- Le chapitre 9 est donc consacré à la modélisation en biogéochimie avec ses différentes facettes : typologie des modèles, échelles d'observation, maillage, changement d'échelle, couplage,...

- Quant au dernier chapitre (chapitre 10), il aborde la question de la mise en place et de la gestion de réseaux d'observation à caractère scientifique. Il présente d'abord un état des lieux et des nécessités indispensables en vue du suivi biogéochimique de deux milieux caractéristiques de la planète :

- pour les milieux aquatiques : les observatoires océaniques (10.1);

- pour les zones terrestres : les réseaux forestiers (10.2).

Mais il traite aussi de la question des observatoires de la radioactivité (10.3).

Tout ceci conduit à proposer une série de conclusions générales ainsi que des recommandations. 